\title{
Non-Markov Processes: The Problem of the Mean First Passage Time
}

\author{
Peter Hänggi and Peter Talkner \\ Institut für Physik, Basel, Switzerland
}

Received August 19, 1981

\begin{abstract}
The theory of the mean first passage time is developed for a general discrete nonMarkov process whose time evolution is governed by a generalized master equation. The mean first passage time is determined by an adjoint matrix $\Omega^{+}$in a form analogous to the Fokker Planck case. The theory is illustrated by two examples: A onedimensional unit step non-Markov process and a non-Markov process with two-step transitions. Explicit expressions for the mean first passage time are derived.
\end{abstract}

\section{Introduction}

The calculation of the mean first passage time of stochastic processes is of great practical interest. For example, its value can be used to estimate switching times in electronic devices and more generally it gives the rate of escape from a domain of attraction. The latter problem occurs in various fields of science as e.g. chemical reaction rates, adsorption on surfaces, optical bistability etc. Results for the mean first passage time have been formulated first for Fokker-Planck processes $[1,2]$. In one dimension, the corresponding equation can be integrated exactly to yield a closed expression for the mean first passage time in terms of the diffusion coefficient and stationary probability $[1,2]$. Quite recently, various authors succeeded in deriving explicit expressions for the mean first passage time of one-dimensional, discrete unit-step (birth and death) Markov processes [3-5].

Compared with a Markov description, the concept of a non-Markovian description presents generally a more realistic modeling of the dynamics of the system under consideration. The reason that a nonMarkov modeling is commonly not used is partly because of its complexity. For time-homogeneous Markov processes it is rather straightforward to obtain dynamic information about the system via an eigenvalue analysis of the probability evolution operator, the time-independent master equation operator. There is no such direct counterpart for non-
Markov processes, because of a time-memory in the corresponding probability evolution operator defining the generalized master equation.

The goal of this work is to derive a practical expression for the mean first passage time of timehomogeneous (discrete) non-Markov processes. This mean first passage time contains nontrivial dynamic information about the system. The main results of the paper can be summarized as follows:

(i) We develop the theory of the mean first passage time for a general discrete one dimensional nonMarkov process whose dynamics is governed by a generalized master equation. The main result, (2.9), for the mean first passage time can be cast in terms of an adjoint operator $\Omega^{+},(2.10)$, into a form analogous to the Fokker-Planck case. The generalization to higher dimensions and to continuous state spaces is immediate.

(ii) As an example of the main result, (2.9), we study a one-dimensional birth and death non-Markov process. We obtain an explicit closed expression for the mean first passage time. The result contains the case of a unit-step Markov process [3-5].

(iii) For a one-dimensional non-Markov process with unit-step transitions and two-step "birth"transitions, i.e. the process does generally not obey detailed balance, we derive an explicit result for the mean first passage time which can be evaluated recursively. 


\section{The Mean First Passage Time}

We consider one-dimensional non-Markov processes $x(t)$ on a discrete state space $\Sigma$. The time rate of change of the single event probability $p_{t}$ is governed by a generalized master equation (GME)

$\dot{p}_{t}=\int_{0}^{t} K_{t-s} p_{s} d s$

where

$K_{t-s} p_{s}(i)=\sum_{j \in \Sigma}\left\{K_{t-s}(i, j) p_{s}(j)-K_{t-s}(j, i) p_{s}(i)\right\}$.

In the theory of (nonequilibrium) statistical mechanics the transition kernels $K_{t-s}(i, j)$ are derived from micro-dynamics. In the theory of continuous time random walks the memory functions $K_{i-s}(i, j)$ are given in terms of a pausing time distribution via an integral equation [6]. Note that the memory functions are not necessarily positive. However, in order for the probabilities $p_{t}(i)$ to remain positive for all times $t$ there must exist certain relationships. In what follows we assume these relationships to be granted and merely consider (2.1) to characterize a certain non-Markov process. We shall consider an interval $I=[0, N]$ of the state space. If initially the random variable assumes the value $j \in I$ the first passage time $\tau(j)$ (a random variable) is the time which elapses before leaving the interval $I$ for the first time. By $f_{t}(i \mid j)$ we denote the probability to be in state $i$ at time $t$ under the condition that the random variable $x$ has started at initial time $t_{0}=0$ at site $j$ and has not left the interval $I$. Then, $F_{t}(j)$

$F_{t}(j)=\sum_{i \in I} f_{t}(i \mid j)$

is the probability that the system is still in interval $I$ at time $t$ given that it started at point $j \in I$. Clearly we have for the initial condition

$f_{0}(i \mid j)=\delta_{i, j} ; \quad F_{0}(j)=1$.

Then, the probability $w_{t}(j) d t$ for the first passage time $\tau(j)$ to lie in the interval $(t, t+d t)$ is given by

$w_{t}(j)=-\frac{\partial}{\partial t} F_{t}(j)$

The mean first passage time $T(j)$ is the average

$T(j)=\int_{0}^{\infty} t w_{t}(j) d t=\int_{0}^{\infty} F_{t}(j) d t$

Thereby we have integrated by parts and have assumed the natural condition that $F_{\infty}(j)$ vanishes ${ }^{\star}$. The dynamics of the conditional probability $f_{t}(i \mid j)$ is gov-

\footnotetext{
* More explicit we have with $F_{\infty}(j)=0$ :

$\lim _{t \rightarrow \infty} t F_{i}(j)=\lim _{t \rightarrow \infty} t \int_{t}^{\infty} w_{t}(j) d t \leqq \lim _{t \rightarrow \infty} \int_{t}^{\infty} t w_{\mathrm{r}}(j) d t=0$

for $T(j)$ being bounded.
}

erned by the GME (2.1) with the boundary conditions at site 0 and $N$ taken into account appropriately. In the following we discuss the case where the interval $I$ is left at the boundary $N$ only. Then, 0 is a reflecting boundary and in order that $f_{t}(i \mid j)$ counts only those trajectories which have not left $I$ the memory functions $K_{t-s}(i \mid j)$ must be modified such that $N$ is an absorbing boundary. Explicitly, we must set $K_{t-s}(i \mid j)=0$ for all $i \in I$ and all $j \geqq N$, that means that there is no back-flow into the interval at site $N$. Cases with other intervals or other boundary conditions can be treated analogously. Thus, we have for the time rate of change of the probability $f_{t}(i \mid j)$ the equation

$\dot{f_{t}}=\int_{0}^{t} \vec{K}_{t-s} f_{s} d s$

where $f_{t}$ denotes the matrix with elements $f_{t}(i \mid j)$ and the matrix $\bar{K}_{t-s}$ is defined in (2.1) with the memory functions properly adjusted at the boundaries as discussed above. In order to arrive at an equation for the probability $F_{t}(j)$ defined in (2.2) we transform (2.6) into the alternative form

$\dot{f}_{t}=\int_{0}^{t} f_{s} \bar{K}_{t-s} d s$

Observing the initial condition $f_{0}=\mathbf{1}$ the equivalence of (2.6) and (2.7) is easily shown with help of the Laplace transformed equations (2.6), (2.7) (see also Ref. 7). By use of the definition of $F_{t}(j)$ one finds

$\dot{F}_{t}=\int_{0}^{t} \bar{K}_{t-s}^{+} F_{s} d s$

where $F_{t}$ is a vector with the components $F_{t}(j), j$ $=0, \ldots, N-1$, and $\bar{K}_{t-s}^{+}$denotes the adjoint of $\bar{K}_{t-s}$. Integrating (2.8) with respect to time $t$ yields in virtue of (2.3) and (2.5)

$\Omega^{+} T=-1$.

Here $T$ is the vector of mean first passage time components $\{T(j)\}$ and $\Omega^{+}$is the matrix defined by

$\Omega^{+}=\int_{0}^{\infty} \bar{K}_{s}^{+} d s$.

The relations (2.9) and (2.10) represent the main result of the paper. Equation (2.9) gives the mean first passage time for a general discrete process on a line. The form in (2.9) is particularly suitable for a computer evaluation (calculation of inverse of the matrix $\Omega^{+}$). The results in (2.9), (2.10) hold for any distribution of jump widths, i.e. the results are not restricted to nearest neighbor transitions only. As a 
special case, (2.9) contains the recent result of Weiss for a continuous time random walk with nearest neighbor transitions [8]. This special result he has derived by use of a different technique ${ }^{+}$. With $\delta$ correlated Markovian transition functions

$K_{t-s}(i, j)=2 \Gamma(i, j) \delta(t-s)$,

i.e. $\Omega_{i j}^{+}=\Gamma_{j i}$, we obtain the mean first passage time for a general discrete Markov process. The corresponding result (2.9) is readily shown to be equivalent to a somewhat formal result derived by Weiss in his 1966 work for Markov processes [9]. Note, that if the time integrals (2.10) of the memory functions are transition probabilities of a Markov process, which then approximates the long time behaviour of the non Markov process under consideration, the mean first passage times of the two processes coincide.

Equations (2.9), (2.10) remain unchanged for a multidimensional discrete non-Markov process if only the matrix of the memory functions is adjusted to the corresponding boundary conditions. Moreover, the results still remain true for processes on a continuous state space with generally nonlocal interactions. The matrix $\bar{K}_{t-s}$ is replaced by an integral operator [10] which again must be adjusted to an absorbing boundary condition. For example, we recover from (2.9) the well known formula for Fokker Planck processes [1-3]

$\Gamma^{+} T=-1, \quad T(x)=0 \quad$ for $x \in \partial \Omega$

where $\Gamma^{+}$is the adjoint Fokker Planck operator and $\partial \Omega$ is the boundary of the domain from which the escape is studied.

Finally, we emphasize that (2.7) has been essential for the derivation of (2.9); Equation (2.7) can be looked upon as the backward equation of a nonMarkov process.

\section{Examples}

\subsection{Birth and Death Non-Markov Processes}

As a first example for the theory developed in Sect. 2 we consider a birth and death non-Markov process

\footnotetext{
+ Weiss's starting point is not the GME, but an equation for absorption probabilities

* We use the relation $\int_{0}^{t} \delta(s) d s=\frac{1}{2}$
}

$$
\begin{aligned}
& \dot{p}_{t}(i)=\int_{0}^{t} d s\left\{K_{t-s}(i, i-1) p_{s}(i-1)\right. \\
& +K_{t-s}(i, i+1) p_{s}(i+1) \\
& \left.-\left[K_{t-s}(i-1, i)+K_{t-s}(i+1, i)\right] p_{s}(i)\right\} \\
& i=0,1, \ldots .
\end{aligned}
$$

This type of a GME occurs for example in the modeling of exciton transport [6]. Because the transition function $K(-1,0)$ equals zero (natural reflecting boundary at site 0 ) the process $x(t)$ satisfies the detailed balance relation

$$
K_{t-s}(i+1, i) \bar{p}(i)=K_{t-s}(i, i+1) \bar{p}(i+1)
$$

with $\bar{p}(i)$ being the stationary probability. Integrating the memory functions over the memory time $s$ we obtain in terms of the matrix elements

$$
\begin{aligned}
& \Omega_{i+1, i}=\int_{0}^{\infty} K_{s}(i+1, i) d s \equiv \Omega_{+}(i) \\
& \Omega_{i-1, i}=\int_{0}^{\infty} K_{s}(i-1, i) d s \equiv \Omega_{-}(i)
\end{aligned}
$$

for the stationary probability $\bar{p}(i)$

$\vec{p}(i)=\bar{p}(0) \prod_{j=1}^{i} \Omega_{+}(j-1) / \Omega_{-}(j)$

With site $x=0$ being a reflecting boundary and site $x=N$ being an absorbing boundary, the matrix $\Omega^{+}$ in (2.9) has the explicit form

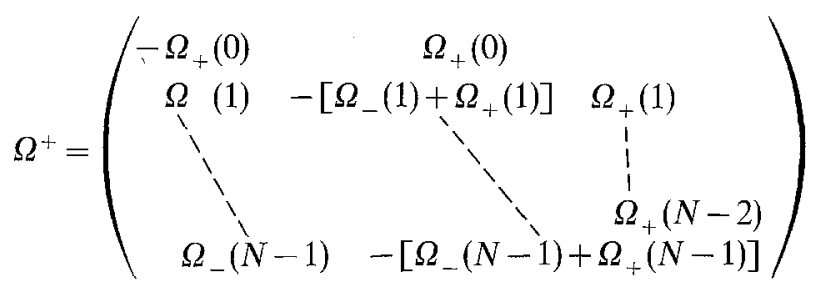

The mean first passage times $T(j), j=0, \ldots N-1$, are given in terms of the inverse of the nonsymmetric, tridiagonal matrix $\Omega^{+}$. In absence of an absorbing state* within the interval $I$, the tridiagonal matrix $\Omega^{+}$is nonsingular and its inverse can be found in terms of recursive methods [11]. In terms of the (non-Markov) matrix $\Omega^{+}$, the problem of calculating $T(j)$ is mathematically equivalent of finding the

\footnotetext{
* For an absorbing state $\{i\}$ we have $\Omega_{+}(i)=\Omega_{-}(i)=0$
} 
mean first passage time for a unit step Markov process. Thus we can make use of the results in [35]: Using the detailed balance relation

$$
\frac{\bar{p}(i)}{\bar{p}(i-1)}=\frac{\Omega_{+}(i-1)}{\Omega_{-}(i)}
$$

an analogous analysis yields the explicit result

$T(j)=\sum_{i=j}^{N-1}\left(\frac{\sum_{n=0}^{i} \bar{p}(n)}{\Omega_{+}(i) \bar{p}(i)}\right) \quad j=0,1, \ldots N-1$.

\subsection{Non-Markov Process with Two-Step Jumps}

Let us consider a non-Markov process $x(t)$ with $\Omega$ matrix "birth" elements

$\Omega_{i+1, i} \equiv \lambda_{i} \quad i=0,1, \ldots$

$\Omega_{i+2, i} \equiv v_{i} \quad i=0,1, \ldots$

and simple "death" elements only

$\Omega_{i-1, i} \equiv \mu_{i} \quad i=1,2, \ldots$

$\Omega_{i-2, i} \equiv 0 \quad i=2,3, \ldots$

In virtue of (2.2), (2.5) we can write for the mean first passage time $T(j)$

$T(j)=\sum_{i=0}^{N-1} \tilde{f}_{i}(j)$

where

$\tilde{f}_{i}(j)=\int_{0}^{\infty} f_{t}(i \mid j) d t$

and $x=0, x=N$ again denote a reflecting and an absorbing boundary respectively. In the following we drop in the function $\tilde{f}_{i}(j)$ the index $j$ which gives the dependence on the starting value $x=j$ at time $t_{0}$ $=0$. From (2.6) we obtain for $\tilde{f}_{i}$ (Laplace transform at $z=0$ ) the set of equations

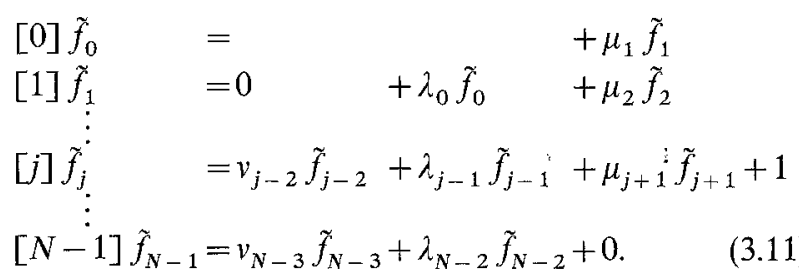

Hereby we introduced the notation

$[i]=\lambda_{i}+\mu_{i}+v_{i}$.

The set of (3.11) can be solved recursively. We introduce

$\tilde{f}_{i}=\theta_{i} \tilde{f}_{0} \quad i=1, \ldots j$

$\tilde{f}_{i}=\theta_{i} \tilde{f}_{0}-b_{i} \quad i=j+1, \ldots N-1$.
Solving for $\tilde{f}_{1}$ we obtain

$\theta_{1}=\frac{[0]}{\mu_{1}} \equiv \frac{a_{0}}{\mu_{1}} ; \quad \theta_{0} \equiv 1$.

The first $j$ equations are satisfied if we write

$\theta_{n+1}=\frac{a_{n}}{\prod_{i=1}^{n+1} \mu_{i}}$

where $\left\{a_{i}\right\}$ satisfies the recursion relation

$a_{i}=[i] a_{i-1}-\mu_{i} \lambda_{i-1} a_{i-2}-\mu_{i} \mu_{i-1} v_{i-2} a_{i-3}$

(3.16a) can be simplified to give

$a_{i}=\left(\lambda_{i}+v_{i}\right) a_{i-1}+\mu_{i} v_{i-1} a_{i-2}$

with

$a_{-1}=1, \quad a_{0}=\left(\lambda_{0}+v_{0}\right)$

The $(j+1)$-st equation gives

$\mu_{j+1} b_{j+1}=1$

and successively

$\mu_{j+2} b_{j+2}=[j+1] b_{j+1}$

$\mu_{j+3} b_{j+3}=[j+2] b_{j+2}-\lambda_{j+1} b_{j+1}-0$

$\mu_{n+1} b_{n+1}=[n] b_{n}-\lambda_{n-1} b_{n-1}-v_{n-2} b_{n-2}$ $n=j+3, \ldots N-1$

The initial value $\tilde{f}_{0}$ is determined from the last equation in (3.11) to be

$\tilde{f}_{0}=b_{N} \prod_{i=1}^{N} \mu_{i} / a_{N-1}=b_{N} / \theta_{N}$.

Therefore, in terms of the functions $\tilde{f}_{i}=\theta_{i} \tilde{f}_{0}-b_{i}$ with $\theta_{i}$ given in (3.15) and $b_{i}$ given by (3.18), we obtain from (3.9) for $T(j)$ the explicit result

$T(j)=\frac{b_{N}}{\theta_{N}} \sum_{i=0}^{j} \theta_{i}+\sum_{i=j+1}^{N-1}\left(\frac{b_{N}}{\theta_{N}} \theta_{i}-b_{i}\right)$.

In case that all $v_{i} \equiv 0$ (birth and death process) we have

$\theta_{i}=\prod_{n=1}^{i} \frac{\Omega_{+}(n-1)}{\Omega_{-}(n)}=\frac{\bar{p}(i)}{\bar{p}(0)}$

and

$$
b_{i}=\bar{p}(i) \sum_{n=j+1}^{i}\left(\bar{p}(n-1) \Omega_{+}(n-1)\right)^{-1},
$$


yielding in virtue of (3.20) the result in (3.7). It should be noted however that $v_{i}=0$, for all $i$ implies always detailed balance whereas the two-step process in (3.8) generally does not obey detailed balance [12]: The result in (3.20) holds independent of any specific form of the $\Omega$-matrix elements $\left\{\lambda_{i}, \mu_{i}, v_{i}\right\}$ and independent of a detailed balance relation.

One of us (P.H.) would like to thank Professor H. Thomas for the hospitality at his Institute. This work was supported by the Swiss National Science Foundation. The authors appreciate the editorial work by Mary Ostendorf at the Physics Department of the University of Illinois at Urbana-Champaign.

\section{References}

1. Pontryagin, L., Andronov, A., Witt, A.: Zh. Eskp. Teor. Fiz. 3, 172 (1933)

2. Stratonovitch, R.L.: Topics in the Theory of Random Noise. Vol. I. New York: Gordon-Breach 1963

3. Weiss, G.H.: Adv. Chem. Phys. 13, 1 (1966)

4. Gillespie, D.T.: Physica A 95, 69 (1979); J. Chem. Phys. 74, $5295(1981)$
5. Seshardi, V., West, B.J., Lindenberg, K.: J. Chem. Phys. 72, 1145 (1980)

6. Kenkre, V.M., Montroll, E.W., Schlesinger, M.F.: J. Stat. Phys. 9, 45 (1973)

7. Grabert, H., Hänggi, P., Talkner, P.: Phys. Lett. 66 A, 255 (1978)

8. Weiss, G.H.: J. Stat. Phys. 24, 587 (1981)

9. Ref. [3], p. 4, Eq. (10)

10. Grabert, H., Talkner, P., Hänggi, P., Thomas, H.: Z. Phys. B Condensed Matter 29, 273 (1978)

11. Wilkinson, J.H.: The Algebraic Eigenvalue Problem. Oxford: Oxford University Press (1969)

12. Haag, G., Hänggi, P.: Z. Phys. B - Condensed Matter 39, 269 (1980)

Peter Talkner

Institut für Physik

Klingelbergstrasse 82

$\mathrm{CH}-4056$ Basel

Switzerland

Peter Hänggi

Department of Physics

Polytechnic Institute of New York

333 Jay Street

Brooklyn, NY 11201

USA 